between July 2004 and June 2005 at a single center in India and had stable graft function 1 month later. Immunosuppression comprised ciclosporin (in $94 \%$ of cases) or tacrolimus, plus mycophenolate mofetil or azathioprine, and prednisolone. The patients were randomized to receive either intraoperative biopsies only (control group; $n=50$ ) or intraoperative biopsies plus protocol biopsies at 1 and 3 months following transplantation (study group; $n=52$ ). Biopsies were also performed in both groups when clinically indicated. The study group had a significantly lower mean level of serum creatinine than the control group at 6 months and 12 months after transplantation $(P=0.0003$ and $P<0.0001$, respectively). In addition, the mean glomerular filtration rate, as estimated by the Modification of Diet in Renal Disease equation, was higher in the study group than in the control group at both time points $(P=0.0036$ at 6 months, and $P=0.0029$ at 12 months). There was no significant difference in the incidence of acute rejection between the two groups.

The authors conclude that early protocol biopsies are useful in patients receiving ciclosporin-based immunosuppression after living-donor kidney transplantation.

Original article Kurtkoti J et al. (2008) The utility of 1- and 3-month protocol biopsies on renal allograft function: a randomized controlled study. Am J Transplant 8: 317-323

\section{UNOS criteria identify candidates for organ donation after cardiac death}

Organs from patients who died more than $60 \mathrm{~min}$ after the withdrawal of life-sustaining treatment (LST) are generally considered unsuitable for transplantation because warm ischemia impairs organ function. Donation after cardiac death is uncommon in the US, partly as a result of the difficulty of prospectively identifying patients who are likely to die within the 60 min window. To facilitate the identification of such individuals, the United Network for Organ Sharing (UNOS) developed a set of criteria in 2002. These criteria have now been validated for the first time, in a prospective study of 505 adults who underwent elective withdrawal of LST at five US hospitals.

In total, $45 \%$ of patients died within $60 \mathrm{~min}$ of the withdrawal of LST. A significant positive association was found between the number of UNOS criteria fulfilled and the likelihood of death within $60 \mathrm{~min}(P<0.001)$. Among the patients with one, two, or three or more UNOS criteria, $52 \%, 65 \%$ and $80 \%$, respectively, died within 60 min of LST withdrawal; the same trend was observed among patients who were classified as 'desirable donor candidates' (i.e. were $<60$ years old, and had serum creatinine $<177 \mu \mathrm{mol} / /[<2 \mathrm{mg} / \mathrm{dl}]$ and no overwhelming sepsis). In the subgroup of patients who fulfilled none of the UNOS criteria, only $29 \%$ died within 60 min of LST withdrawal.

These results, say the authors, validate the UNOS criteria, and indicate that patients who fulfill at least two criteria are optimal candidates for donation after cardiac death; however, when a $50 \%$ failure rate is acceptable, patients with a single criterion should be considered.

Original article DeVita MA et al. (2008) Donors after cardiac death: validation of identification criteria (DVIC) study for predictors of rapid death. Am J Transplant 8: 432-441

\section{Laparoscopic living-donor nephrectomy: shorter operating time for right kidney}

Whether the right or left kidney is preferable in living-donor nephrectomy is the subject of much debate. In view of evidence that prolonged pneumoperitoneum during the procedure adversely affects kidney function, Minnee et al. conducted a prospective, randomized, singlecenter trial to compare operating times for left-sided and right-sided donor nephrectomy.

The study, which took place from April 2002 until September 2006, included 60 consecutive adults scheduled for transperitoneal handassisted laparoscopic donor nephrectomy; 31 individuals underwent right-sided nephrectomy and 29 underwent left-sided nephrectomy. Donors were required to have kidneys with symmetrical function and anatomy. The primary end point-median operating time-was $180 \mathrm{~min}$ for left-sided nephrectomy compared with $150 \mathrm{~min}$ for right-sided nephrectomy $(P=0.021)$. The median duration of warm ischemia was $3 \mathrm{~min}$ in both groups. No conversions to open nephrectomy were necessary in either group. No major complications occurred during or after surgery, and median hospital stay was 4 days for both groups. All patients had recovered to a preoperative quality-of-life score by 3-6 months after surgery. In the recipients, the rate of acute rejection did not differ significantly between 